Annals of Pure and Applied Mathematics

Vol. 17, No. 1, 2018, 107-112

ISSN: 2279-087X (P), 2279-0888(online)

Published on 10 May 2018

www.researchmathsci.org

DOI: http://dx.doi.org/10.22457/apam.v17n1a12

Annals of

Pure and Applied

Mathematics

\title{
Two New Arithmetic-Geometric ve-degree Indices
}

\author{
V.R.Kulli
}

Department of Mathematics

Gulbarga University, Gulbarga 585106, India

e-mail: vrkulli@gmail.com

Received 7 April 2018; accepted 9 May 2018

Abstract. Recently, the ve-degree concept is defined in Graph Theory. We introduce the arithmetic-geometric ve-degree index and multiplicative arithmetic-geometric ve-degree index of a molecular graph. In this paper, we compute these ve-degree topological indices for some networks such as dominating oxide networks and regular triangulate oxide networks.

Keywords: arithmetic-geometric ve-degree index, multiplicative arithmetic-geometric ve-degree index, dominating oxide network, regular triangulate oxide network.

\section{AMS Mathematics Subject Classification (2010): 05C05, 05C12, $05 C 35$}

\section{Introduction}

Let $\mathrm{G}$ be a finite, simple connected graph with vertex set $V(G)$ and edge set $E(G)$. The degree $d_{G}(v)$ of a vertex $v$ is the number of vertices adjacent to $v$. The set of all vertices which adjacent to $v$ is called the open neighborhood of $v$ and denoted by $N(v)$. The closed neighborhood set of $v$ is the set $N[v]=N(v) \cup\{v\}$. Let $S_{v}$ denote the sum of the degrees of all vertices adjacent to a vertex $v$.

In [2], Chellali et al. defined the ve-degree concept in graph theory as follows:

The ve-degree $d_{v e}(v)$ of a vertex $v$ in a graph $G$ is the number of different edges that incident to any vertex from the closed neighborhood of $v$.

Chemical Graph Theory is a branch of Graph Theory whose focus of interest is to finding topological indices of chemical graphs, which correlate well with chemical properties of the chemical molecules. Numerous topological indices have been considered in Theoretical chemistry, especially in QSAR and QSPR research, see [1].

Recently, Ediz [8] defined the ve-degree geometric-arithmetic index of a connected graph $G$ and it is defined as

$$
G A_{v e}(G)=\sum_{u v \in E(G)} \frac{2 \sqrt{d_{v e}(u) d_{v e}(v)}}{d_{v e}(u)+d_{v e}(v)} .
$$
follows:

We now introduce the arithmetic-geometric ve-degree index of a graph as

The arithmetic-geometric ve-degree index of a graph $G$ and it is defined as 


$$
A G_{v e}(G)=\sum_{u v \in E(G)} \frac{d_{v e}(u)+d_{v e}(v)}{2 \sqrt{d_{v e}(u) d_{v e}(v)}} .
$$

The multiplicative geometric-arithmetic ve-degree index was defined by Kulli [5] and defined as

$$
G A_{v e} I I(G)=\prod_{u v \in E(G)} \frac{2 \sqrt{d_{v e}(u) d_{v e}(v)}}{d_{v e}(u)+d_{v e}(v)} .
$$

Motivated by the definition of the multiplicative geometric-arithmetic ve-degree index and its applications, we introduce the multiplicative arithmetic-geometric ve-degree index of a graph as follows:

The multiplicative arithmetic-geometric ve-degree index of a graph $G$ is defined as

$$
A G_{v e} I I(G)=\prod_{u v \in E(G)} \frac{d_{v e}(u)+d_{v e}(v)}{2 \sqrt{d_{v e}(u) d_{v e}(v)}} .
$$

Recently, some topological indices were studied, for example, in $[3,4,6$, $7,9,10,11]$.

We consider the families of dominating oxide networks and regular triangulate oxide networks [8]. In this paper, the arithmetic-geometric ve-degree index and multiplicative arithmetic-geometric ve-degree index of dominating oxide networks and regular triangulate oxide networks are determined.

\section{Results for dominating oxide networks}

The family of dominating oxide networks is symbolized by $\operatorname{DOX}(n)$. The molecular structure of a dominating oxide network is presented in Figure 1.

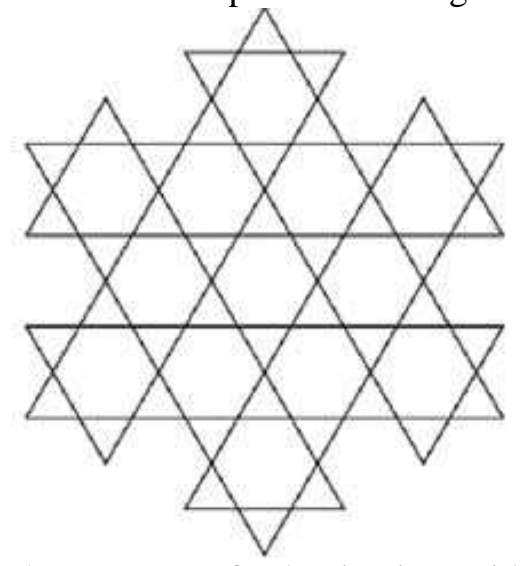

Figure 1: The structure of a dominating oxide network

In [8], Ediz obtained the partition of the edges with respect to their sum degree of end vertices of dominating oxide networks in Table 1.

\begin{tabular}{ccccccc}
\hline$\left(S_{u}, S_{v}\right)$ & $(8,12)$ & $(8,14)$ & $(12,12)$ & $(12,14)$ & $(14,16)$ & $(16,16)$ \\
\hline $\begin{array}{c}\text { Number of } \\
\text { edges }\end{array}$ & $12 n$ & $12 n-12$ & 6 & $12 n-12$ & $24 n-24$ & $54 n^{2}-114 n+60$ \\
\hline \multicolumn{5}{c}{ Table 1 } \\
& \multicolumn{7}{c}{108}
\end{tabular}


Two New Arithmetic-Geometric ve-degree Indices

Also he obtained the ve-degree partition of the end vertices of edges for dominating oxide networks in Table 2.

\begin{tabular}{ccccccc}
\hline $\begin{array}{c}\left(d_{v e}(u),\right. \\
\left.d_{v e}(v)\right)\end{array}$ & $(7,10)$ & $(7,12)$ & $(10,10)$ & $(10,12)$ & $(12,14)$ & $(14,14)$ \\
\hline $\begin{array}{c}\text { Number of } \\
\text { edges }\end{array}$ & $12 n$ & $12 n-12$ & 6 & $12 n-12$ & $24 n-24$ & $54 n^{2}-114 n+60$ \\
\hline
\end{tabular}

Table 2: The ve-degree of the end vertices of edges for DOX networks

In the following theorem, we compute the arithmetic-geometric ve-degree index of $\operatorname{DOX}(n)$.

Theorem 1. The arithmetic-geometric ve-degree index of a dominating oxide network $\operatorname{DOX}(n)$ is

$$
\begin{aligned}
A G_{v e}(D O X(n)) & =54 n^{2}+\left(\frac{102}{\sqrt{70}}+\frac{57}{\sqrt{21}}+\frac{66}{\sqrt{30}}+\frac{156}{\sqrt{42}}-114\right) n \\
& -\left(\frac{57}{\sqrt{21}}+\frac{66}{\sqrt{30}}+\frac{156}{\sqrt{42}}-66\right) .
\end{aligned}
$$

Proof: Let $G$ be the graph of a dominating oxide network $D O X(n)$. By using equation (1) and Table 2, we deduce

$$
\begin{aligned}
& A G_{v e}(D O X(n))=\sum_{u v \in E(G)} \frac{d_{v e}(u)+d_{v e}(v)}{2 \sqrt{d_{v e}(u) d_{v e}(v)}} \\
& =\left(\frac{7+10}{2 \sqrt{7 \times 10}}\right) 12 n+\left(\frac{7+12}{2 \sqrt{7 \times 12}}\right)(12 n-12)+\left(\frac{10+10}{2 \sqrt{10 \times 10}}\right) 6 \\
& +\left(\frac{10+12}{2 \sqrt{10 \times 12}}\right)(12 n-12)+\left(\frac{12+14}{2 \sqrt{12 \times 14}}\right)(24 n-24) \\
& +\left(\frac{14+14}{2 \sqrt{14 \times 14}}\right)\left(54 n^{2}-114 n+60\right) \\
& =54 n^{2}+\left(\frac{102}{\sqrt{70}}+\frac{57}{\sqrt{21}}+\frac{66}{\sqrt{30}}+\frac{156}{\sqrt{42}}-114\right) n \\
& -\left(\frac{57}{\sqrt{21}}+\frac{66}{\sqrt{30}}+\frac{156}{\sqrt{42}}-66\right) .
\end{aligned}
$$

In the following theorem, we compute the multiplicative arithmetic-geometric ve-degree index of $\operatorname{DOX}(n)$.

Theorem 2. The multiplicative arithmetic-geometric ve-degree index of a dominating oxide network $\operatorname{DOX}(n)$ is 
$A G_{v e} I I(D O X(n))=\left(\frac{17}{2 \sqrt{70}}\right)^{12 n} \times\left(\frac{19}{4 \sqrt{21}}\right)^{12 n-12} \times\left(\frac{11}{2 \sqrt{30}}\right)^{12 n-12} \times\left(\frac{13}{2 \sqrt{42}}\right)^{24 n-24}$.

Proof: Let $G$ be the graph of a dominating oxide network $D O X(n)$. By using equation (2) and Table 2, we deduce

$$
\begin{aligned}
& A G_{v e} I I(D O X(n))=\prod_{u v \in E(G)} \frac{d_{v e}(u)+d_{v e}(v)}{2 \sqrt{d_{v e}(u) d_{v e}(v)}} \\
& =\left(\frac{7+10}{2 \sqrt{7 \times 10}}\right)^{12 n} \times\left(\frac{7+12}{2 \sqrt{7 \times 12}}\right)^{12 n-12} \times\left(\frac{10+10}{2 \sqrt{10 \times 10}}\right)^{6} \\
& \times\left(\frac{10+12}{2 \sqrt{10 \times 12}}\right)^{12 n-12} \times\left(\frac{12+14}{2 \sqrt{12 \times 14}}\right)^{24 n-24} \\
& \left.\times\left(\frac{14+14}{2 \sqrt{14 \times 14}}\right)^{54 n^{2}-114 n+60}\right)^{12 n} \times\left(\frac{19}{4 \sqrt{21}}\right)^{12 n-12} \times\left(\frac{11}{2 \sqrt{30}}\right)^{12 n-12} \times\left(\frac{13}{2 \sqrt{42}}\right)^{24 n-24} .
\end{aligned}
$$

\section{Results for regular triangulate oxide networks $R T O X(n)$}

The family of regular triangulate oxide networks is denoted by $\operatorname{RTOX}(n), n \geq 3$. The molecular structure of a regular triangulate oxide network is shown in Figure 2.

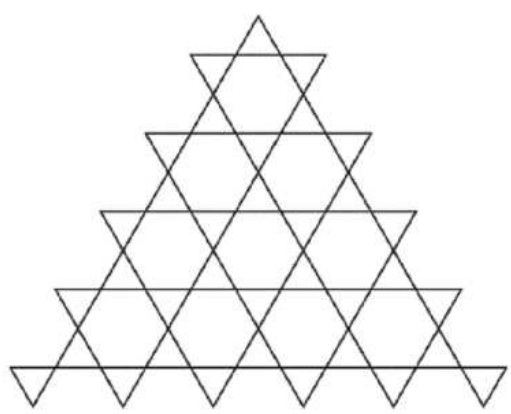

Figure 2: The structure of a regular triangulate oxide network

Ediz [8] obtained the partition of the edges with respect to their sum degree of end vertices of regular triangulate oxide networks in Table 3.

\begin{tabular}{cccccccccc}
\hline$(S u, S v)$ & $(6,6)$ & $(6,12)$ & $(8,12)$ & $(8,14)$ & $(12,12)$ & $(12,14)$ & $(14,14)$ & $(14,16)$ & $(16,16)$ \\
\hline $\begin{array}{c}\text { Number of } \\
\text { edges }\end{array}$ & 2 & 4 & 4 & $6 n-8$ & 1 & 6 & $6 n-9$ & $6 n-12$ & $3 n 2-12 n+12$ \\
& & & & & & & & \\
\hline
\end{tabular}

\section{Table 3}

Also he obtained the ve-degree partition of the end vertices of edges for regular triangulate oxide networks in Table 4. 
Two New Arithmetic-Geometric ve-degree Indices

\begin{tabular}{cccccccccc}
\hline $\begin{array}{l}\text { (dve }(u), \\
\text { dve }(v))\end{array}$ & $(5,5)$ & $(5,10)$ & $(7,10)$ & $(7,12)$ & $(10,10)$ & $(10,12)$ & $(12,12)$ & $(12,14)$ & $(14,14)$ \\
\hline $\begin{array}{c}\text { Number of } \\
\text { edges }\end{array}$ & 2 & 4 & 4 & $6 n-8$ & 1 & 6 & $6 n-9$ & $6 n-12$ & $3 n 2-$ \\
\end{tabular}

Table 4: The ve-degree of the end vertices of edges for RTOX networks

In the following theorem, we compute the arithmetic-geometric ve-degree index of $\operatorname{RTOX}(n)$.

Theorem 3. The arithmetic-geometric ve-degree index of a regular triangulate oxide network $\operatorname{RTOX}(n)$ is

$$
A G_{v e}(\operatorname{RTOX}(n))=3 n^{2}+\left(\frac{57}{2 \sqrt{21}}+\frac{39}{\sqrt{42}}-6\right) n+\left(\frac{30}{\sqrt{50}}+\frac{34}{\sqrt{70}}-\frac{38}{\sqrt{21}}+\frac{33}{\sqrt{30}}-\frac{78}{\sqrt{42}}+6\right) \text {. }
$$

Proof: Let $G$ be the graph of a regular triangulate oxide network RTOX $(n)$. By using equation (1) and Table 4, we derive

$$
\begin{aligned}
A G_{v e}( & R T O X(n))=\sum_{u v \in E(G)} \frac{d_{v e}(u)+d_{v e}(v)}{2 \sqrt{d_{v e}(u) d_{v e}(v)}} \\
& =\left(\frac{5+5}{2 \sqrt{5 \times 5}}\right) 2+\left(\frac{5+10}{2 \sqrt{5 \times 10}}\right) 4+\left(\frac{7+10}{2 \sqrt{7 \times 10}}\right) 4+\left(\frac{7+12}{2 \sqrt{7 \times 12}}\right)(6 n-8) \\
& +\left(\frac{10+10}{2 \sqrt{10 \times 10}}\right) 1+\left(\frac{10+12}{2 \sqrt{10 \times 12}}\right) 6+\left(\frac{12+12}{2 \sqrt{12 \times 12}}\right)(6 n-9) \\
& +\left(\frac{12+14}{2 \sqrt{12 \times 14}}\right)(6 n-12)+\left(\frac{14+14}{2 \sqrt{14 \times 14}}\right)\left(3 n^{2}-12 n+12\right) \\
& =3 n^{2}+\left(\frac{57}{2 \sqrt{21}}+\frac{39}{\sqrt{42}}-6\right) n+\left(\frac{30}{\sqrt{50}}+\frac{34}{\sqrt{70}}-\frac{38}{\sqrt{21}}+\frac{33}{\sqrt{30}}-\frac{78}{\sqrt{42}}+6\right) .
\end{aligned}
$$

In the following theorem, we compute the multiplicative arithmetic-geometric ve-degree index of $\operatorname{RTOX}(n)$.

Theorem 4. The multiplicative arithmetic-geometric ve-degree index of a regular triangulate oxide network $\operatorname{RTOX}(n)$ is

$$
A G_{v e} I I(\operatorname{RTOX}(n))=\left(\frac{15}{2 \sqrt{50}}\right)^{4} \times\left(\frac{17}{2 \sqrt{70}}\right)^{4} \times\left(\frac{19}{4 \sqrt{21}}\right)^{6 n-8} \times\left(\frac{11}{2 \sqrt{30}}\right)^{6} \times\left(\frac{13}{2 \sqrt{42}}\right)^{6 n-12} .
$$

Proof: Let $G$ be the graph of a regular trianglate oxide network $R T O X(n)$. By using equation (2) and Table 4, we derive

$$
A G_{v e} I I(R T O X(n))=\prod_{u v \in E(G)} \frac{d_{v e}(u)+d_{v e}(v)}{2 \sqrt{d_{v e}(u) d_{v e}(v)}}
$$


V.R.Kulli

$$
\begin{aligned}
& =\left(\frac{5+5}{2 \sqrt{5 \times 5}}\right)^{2} \times\left(\frac{5+10}{2 \sqrt{5 \times 10}}\right)^{4} \times\left(\frac{7+10}{2 \sqrt{7 \times 10}}\right)^{4} \times\left(\frac{7+12}{2 \sqrt{7 \times 12}}\right)^{6 n-8} \times\left(\frac{10+10}{2 \sqrt{10 \times 10}}\right)^{1} \\
& \times\left(\frac{10+12}{2 \sqrt{10 \times 12}}\right)^{6} \times\left(\frac{12+12}{2 \sqrt{12 \times 12}}\right)^{6 n-9} \times\left(\frac{12+14}{2 \sqrt{12 \times 14}}\right)^{6 n-12} \times\left(\frac{14+14}{2 \sqrt{14 \times 14}}\right)^{3 n^{2}-12 n+12} \\
& =\left(\frac{15}{2 \sqrt{50}}\right)^{4} \times\left(\frac{17}{2 \sqrt{70}}\right)^{4} \times\left(\frac{19}{4 \sqrt{21}}\right)^{6 n-8} \times\left(\frac{11}{2 \sqrt{30}}\right)^{6} \times\left(\frac{13}{2 \sqrt{42}}\right)^{6 n-12} .
\end{aligned}
$$

Acknowledgement. The author is thankful to the referee for useful suggestions.

\section{REFERENCES}

1. I.Gutman and O.E. Polansky, Mathematical Concepts in Organic Chemistry, Springer, Berlin (1986).

2. M.Chellali, T.W.Hynes, S.T.Hedetniemi and T.W. Lewis, On ve-degrees and evdegrees in graphs, Discrete Mathematics, 340(2) (2017) 31-38.

3. B.Sahil and S.Ediz, On ev-degree and ve-degree topological indices, Iranian Journal of Mathematical Chemistry, in press.

4. V.R.Kulli, On ve-degree indices and their polynomials of dominating oxide networks, submitted.

5. V.R.Kulli, Multiplicative connectivity ve-degree indices of dominating oxide and regular triangulate oxide networks, submitted.

6. V.R.Kulli, General multiplicative ve-degree indices of dominating oxide and regular triangulate oxide networks, submitted.

7. S.Ediz, Predicting some physicochemical properties of octane isomers: A topological approach using ve-degree and ve-degree Zagreb indices, International Journal of System Science and Applied Mathematics, 2(2017) 87-92.

8. S.Ediz, On ve-degree molecular topological properties of silicate and oxygen networks, Int. J. Computing Science and Mathematics, 9(1) (2018) 1-12.

9. V.R.Kulli, Two new multiplicative atom bond connectivity indices, Annals of Pure and Applied Mathematics, 13(1) (2017) 1-7.

10. V.R.Kulli, New arithmetic geometric indices, Annals of Pure and Applied Mathematics,13(2) (2017) 165

11. V.R.Kulli, The sum connectivity Revan index of silicate and hexagonal networks, Annals of Pure and Applied Mathematics, 14 (3) (2017) 401-406. 